\title{
Judging the value of ammonia measurement on lactulose dosing: Apples and oranges?
}

\author{
Christopher F Rose PhD', Rajiv Jalan MD²
}

KEYWORDS: ammonia; hepatic encephalopathy; lactulose

\author{
Author Affiliation \\ ${ }^{1}$ Hepato-Neuro Laboratory, CRCHUM, Université de Montréal, Montréal, Québec, Canada; ${ }^{2}$ University College London- \\ Liver Failure Group, Institute for Liver and Digestive Health London, London, United Kingdom
}

Correspondence: Christopher F Rose, Hepato-Neuro Laboratory, Centre Hospitalier de l'Université de Montréal Medicine, 900 rue St-Denis, Montréal, Québec H2X 0A1 Canada. Telephone: 514-890-8000, ext 35739. E-mail: christopher.rose@umontreal.ca

Haj M, Rockey DC. Ammonia Levels Do Not Guide Clinical Management of Patients With Hepatic Encephalopathy Caused by Cirrhosis. Am J Gastroenterol. 2020;115(5):723-728.

The central role of ammonia in the pathogenesis of hepatic encephalopathy (HE) is well recognized (1). However, the measurement of ammonia for the management of HE is controversial. Haj and Rockey attempted to clarify this dispute (2) with a retrospective study in a large number of patients in a United States (US) single-centre hospital. They found the dose of lactulose administered to patients did not differ in those with either elevated or normal ammonia, concluding that ammonia levels do not guide therapy in the management of HE. This conclusion needs to be examined carefully.

In the study, $46 \%$ (551 patients) of the patients who were hospitalized with overt HE had ammonia measured. From these measurements, $60 \%$ were defined as being hyperammonemic using a cut-off for blood ammonia of $>72 \mathrm{uM}$. This requires discussion. First, there is a strong selection bias since $54 \%$ of patients diagnosed with overt HE did not have their ammonia measured, and no reason was provided. Second, $72 \mu \mathrm{M}$ cut-off needs to be justified; this level of ammonia is exceedingly high to be defined as normal since it was recently demonstrated that an ammonia value of $79.5 \mu \mathrm{M}$ is independently associated with death within the next 28 days (3). Therefore, $40 \%$ patients with 'normal' ammonia levels is an overstatement and most likely inaccurate, as the diagnosis of $\mathrm{HE}$ is not compatible with normal ammonia levels (4). Third, measuring ammonia is delicate, and the acquisition of samples for ammonia measurement, as well as transport and analysis details, were not declared. Sample transport must be on ice to avoid erroneous values, and measurements must be made within 30-60 min (5). In addition, the reference range of ammonia, as well as biochemical analyzer and assay used, needs to be provided. The aforementioned particulars can impact ammonia quantitatively, which leaves the presented ammonia data unreliable.

The primary outcome of their retrospective study was the total amount of lactulose given for

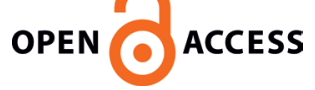


the management of HE within the first 48 hours (time when 3-4 movements per day are routinely achieved). The authors concluded that there was no correlation between the amount of lactulose given and levels of ammonia, signifying that a higher blood ammonia did not entail a more aggressive dose of lactulose. However, it is unclear what guided the dosing of lactulose. Nevertheless, it can be speculated that more lactulose was administered since either constipation was not resolved or HE was persisting. Furthermore, independent of whether ammonia was high or not, or of the precipitating event, all patients received similar volumes of lactulose. This is curious as, in clinical practice, one would primarily use lactulose for patients with constipation and gastrointestinal bleeding and considerably less in those presenting with diarrhea, severe dehydration, renal dysfunction, or infection (4). Remarkably, in patients for whom infection was the precipitating factor $(42 \%)$, lactulose was administered. Despite this, the authors focus on incomplete and potentially inaccurate measurements of ammonia to conclude that the dose of lactulose administered was not influenced by blood ammonia levels.

Finally, lactulose is a non-absorbable disaccharide that acts as an osmotic laxative to treat constipation, a precipitating factor of HE. In North America, lactulose and rifaximin are currently the only available options for the treatment of HE. Lactulose was never approved by the US Food and Drug Administration (FDA), and although used widely, there is no clear regulatory guidance on the indications and doses to be used. Rifaximin was approved by the FDA for the recurrence of HE as secondary prophylaxis and not for the treatment of an acute episode of HE-hospitalization (4). There is only very low-quality data on dose-response of ammonia reduction or HE improvement with lactulose dosing. Furthermore, only a handful of trials exist comparing lactulose with placebo on ammonia levels in the acute setting.

According to Haj and Rockey's study, it is clear lactulose was not used to lower ammonia, as lactulose was administered irrelevant of blood ammonia levels. An efficient ammonia reducing agent remains an unmet care gap, which would mandate ammonia measurements, improve management (differential diagnosis), and guide appropriate treatment for patients suffering from HE. Furthermore, blood ammonia levels have recently been recognized as prognostic and predictive biomarkers for the risk of developing overt HE $(3,6-8)$. This strengthens the value of monitoring and treating blood ammonia.

Based upon an unsound, poorly designed retrospective study, we believe Haj and Rockey's conclusion that ammonia levels do not change the management of these patients is potentially flawed. Prospective studies are needed to thoroughly appreciate the comprehensive role ammonia plays in the spectrum of HE.

CONTRIBUTIONS: Conceptualization, CFR, RJ; Writing - Original Draft, CFR, RJ; Writing - Review and Editing, CFR, RJ.

ETHICS APPROVAL: N/A

INFORMED CONSENT: N/A

REGISTRY AND REGISTRATION NO. OF THE STUDY/

TRIAL: N/A

FUNDING: No funding was received for this work. DISCLOSURES: Christopher Rose has research collaborations with Mallinckrodt Pharma and is an advisor for Axcella, Morphocell Technologies, Sana Biotechnologies, Horizon Therapeutics, and Lupin Pharma. Rajiv Jalan is the inventor of OPA, which has been patented by UCL and licensed to Mallinckrodt Pharma. He is also the founder of Yaqrit Discovery, a spinout company from University College London, Hepyx Limited, and Cyberliver. He has research collaborations with Takeda and Yaqrit Discovery.

\section{REFERENCES}

1. Rose CF, Amodio P, Bajaj JS, et al. Hepatic encephalopathy: novel insights into classification, pathophysiology and therapy. J Hepatol. 2020; 73(6):1526-47. https://doi.org/10.1016/j.jhep. 2020.07.013. Medline:33097308

2. Haj M, Rockey DC.Ammonia levels do not guide clinical management of patients with hepatic encephalopathy caused by cirrhosis. Am J Gastroenterol. 2020;115(5):723-8. https:/ /doi. org/10.14309/ajg.0000000000000343. Medline: 31658104

3. Shalimar, Sheikh MF, Mookerjee RP, Agarwal B, Acharya SK, Jalan R. Prognostic role of ammonia in patients with cirrhosis. Hepatology. 2019;70(3):982-94. https://doi.org/10.1002/ hep.30534. Medline:30703853

4. Vilstrup H, Amodio P, Bajaj J, et al. Hepatic encephalopathy in chronic liver disease: 2014 Practice Guideline by the American Association 
for the Study of Liver Diseases and the European Association for the Study of the Liver. Hepatology. 2014;60(2):715-35. https://doi.org/ 10.1002/hep.27210. Medline:25042402

5. Bajaj JS, Lauridsen M, Tapper EB, et al. Important unresolved questions in the management of hepatic encephalopathy: an ISHEN consensus. Am. J Gastroenterol. 2020;115(7):989-1002. https://doi.org/ 10.14309/ajg.0000000000000603. Medline:32618647

6. Senzolo M, Zarantonello L, Formentin C, et al. Predictive value of induced hyperammonaemia and neuropsychiatric profiling in relation to the occurrence of post-TIPS hepatic encephalopathy. Metab Brain Dis. 2019;34(6):1803-12. https:// doi.org/10.1007/s11011-019-00490-5. Medline: 31506797

7. Patwardhan VR, Jiang ZG, Risech-Neiman Y, et al. Serum ammonia is associated with transplantfree survival in hospitalized patients with acutely decompensated cirrhosis. J. Clin Gastroenterol. 2016;50(4):345-50. https://doi.org/10.1097/MCG. 0000000000000443. Medline:26565968

8. VierlingJM,MokhtaraniM,BrownRS, etal.Fasting blood ammonia predicts risk and frequency of hepatic encephalopathy episodes in patients with cirrhosis. Clin Gastroenterol Hepatol. 2016; 14(6):903-6.e1. https://doi.org/10.1016/j.cgh. 2015.11.018. Medline:26707685 